\title{
La asociatividad como fenómeno evolutivo, análisis de casos colombianos.
}

\section{ASSOBIATIVITY IV THE EVOLUTION PIIENOMEVON- AMAIVSIS OF GASES IW GOLMBIA}

FECHA DE RECEPCIÓN: 19 de febrero de 2010 FECHA DE APROBACIÓN: 29 de abril de 2010 Pp. 100-111.

\section{Juan Gonzalo Castellanos M.*}

* Ingeniero Industrial-Universidad

Distrital Francisco José de Caldas; Especialización Departament of Health and Social Securuty, Hospital

Engineering; investigador Universidad EAN.

Ha sido docente, consultor e investigador de universidades como: Universidad de La Sabana,

Pontificia Universidad Javeriana, Fundación Universidad Autónoma de Colombia, Fundación Andina para el Desarrollo Tecnológico y Social,

Universidad Externado de Colombia. Autor de diferentes libros en el área de Asociatividady de artículos publicados en revistas. Director y autor de numerosos proyectos de investigación. 


\section{RESUMEN}

$L$ a asociatividad, expresada en la conformación de redes de negocios o clústers localizados de empresas en una industria específica, ha sido estudiada por numerosos autores en el mundo desarrollado. Para Latinoamérica y para las PyMEs representa una ventaja importante frente a los retos de productividad y competitividad y a oportunidades en nichos de mercado que se abren en el concierto global; especialmente, en la forma de establecer cooperaciones, colaboraciones y competencias, en aspectos como el mercadeo y la innovación.

Para analizar el estado de estas experiencias a través de casos comparativos, es necesario tener como referente un enfoque evolutivo de la situación, especialmente de la innovación. (Nelson, 1994; Melcalfe,1994:95; Lundvall;1992) han planteado estrategias evolutivas de cambio y perspectivas sistémicas para la innovación, que proporcionan un marco de análisis. De la misma manera, la incorporación de la teoría de sistemas y de redes al ámbito de la microeconomía (Potts, 2008) y la propuesta de políticas evolutivas de ciencia y tecnología que acompañan las prácticas de la innovación (Teubal;2006), facilitan la descripción de su evolución.

Esta investigación, presenta - a través del análisis comparativo de dos experiencias de aglomeración industrial en Colombia que se construyen actualmente- una perspectiva de la evolución de la asociatividad y plantea etapas de dicha evolución, proponiendo que ésta se alcanza por fases que muestran características más o menos definidas.

Esta propuesta de etapas, tiene consecuencias en la conformación de las aglomeraciones industriales que son jalonadas por empresas tractoras, desarrolladas por entes facilitadores o impulsadas espontáneamente por las propias empresas. También tiene consecuencias en la evolución de la política de ciencia, tecnología e innovación.

\section{ABSTRACT}

A ssociativity, seen in the formation of business networks or clusters located in companies of a specific industrial sector, has been studiedby various authors from the developed world. For Latinamerica and for PYMES represent an important advantage as a way of grouping them facing the productive and competitive challenges and the opportunities in marketing niches that are open in globalization. Specifically, they represent a way for establishing cooperation and collaboration trends, competing in the market and innovation fields.

For this analysis the ongoing state of experiences through comparative cases, it is necessary to take the evolution focus as a referent for this situation, especially for the innovation phenomenon. Nelson-1994; Melcalfe- 1994; Lundvall-1992, have stated the evolution strategies of change and systematic perspectives for the innovation that provides a framework for analysis. Similarly, the inclusion of a systems theory and networks to the field of microeconomy, Potts-2008, and the proposed policies of science and technology that go together with innovation practices, Teubal-2006, definitely facilitate the description of this evolution.

This research paper shows a comparative analysis of two experiences on industrial grouping in Colombia that are currently being built, a perspective in the evolution of associativity and suggests the steps of such evolution, proposing that associativity reaches the phases that show more or less definite features.

This proposal of steps has consequences on industrial groupings that are clearly represented by tractor companies, developed by facilitating agents or created by such companies. Besides, it describes effects on the evolution of polices for science, technology and innovation.

\section{RÉSUMÉ}

$\mathrm{L}$ 'associativité désignée comme la conformation de réseaux d'affaires ou pôles de compétences d'entreprises localisés dans une industrie spécifique a été l'objet de nombreuses études dans les pays développés. Pour l'Amérique du Sud et pour les PME-PMI, l'associativité représente un avantage important comme forme de regroupement face aux défis de productivité et aux opportunités de niche de marché qui se créent au niveau mondial, et plus spécialement comme forme d'établissement de relations de coopération et de collaboration sur le plan marketing et de l'innovation.

Pour analyser l'état de ces expériences au travers de cas comparatifs, il est nécessaire d'avoir pour référence une approche évolutive de la situation et plus particulièrement de celle de l'innovation. Nelson-1994; Melcalfe-1994,95; Lundvall-1992 ont mis au point des stratégies évolutives de changement et de perspectives systémiques pour l'innovation qui définissent un cadre d'analyse. De la même manière, le rattachement à la théorie informatique et des réseaux au domaine de la microéconomie Potts-2008 et la proposition de politiques évolutives en science et technologie accompagnent les pratiques de l'innovation. Teubal-2006 en facilite la description de leur évolution. Ce travail de recherche présente au travers de l'analyse comparative de deux expériences de regroupement industriel en devenir en Colombie, une perspective de l'évolution de l'associativité et jalonne les étapes de cette évolution en proposant que l'associativité soit atteinte par des phases révélant des caractéristiques plus ou moins définies. Ces phases d'étape ou de transition ont des conséquences sur la conformation des regroupements industriels qui sont jalonnés par des entreprises leaders développés par des entités facilitatrices ou impulsés spontanément par les entreprises elles mêmes. Cela a également des conséquences sur l'évolution de la politique technologique, scientifique et de l'innovation.
Palabras claves

Asociatividad.

Evolución

Innovación.

Innovation

Política

Ciencia y tecnología

Aglomeración

\section{Key Words}

Associativity

Evolution

Networks

Innovation

Policies

Science-Technology

Grouping

\section{Mots Clefs}

Associativité

Évolution

Réseaux

Innovation

Politique

Science et Technologie

Regroupement 


\section{Introducción}

a asociatividad, expresada en la conformación de redes de negocios o clusters localizados de empresas en una industria específica ha sido estudiada por numerosos autores en el mundo desarrollado.

Para Latinoamérica y para las PyMEs representa una ventaja importante como forma de aglomeración frente a retos de productividad y competitividad y a oportunidades en nichos de mercado que se abren y cierran en el concierto global.

La asociatividad, es de vital importancia en el panorama empresarial y se acentúa en el caso de PyMEs, por la necesidad que tienen estas empresas de competir en mercados nacionales e internacionales con empresas de otras regiones en vías de desarrollo, las cuales ya están organizadas y son altamente competitivas, como es el caso de India, País Vasco, algunas zonas de Argentina, Chile, Brasil y México. Los ejercicios o casos que se analizan en este documento, se realizaron orientados hacia la consolidación permanente de una aglomeración o clúster de empresas siguiendo el camino de otros países, con cimientos adquiridos en la interacción social para la producción.

La universidad se ubica como un ente de soporte en los dos casos que se analizan, con el propósito de apoyar la construcción de relaciones productivas en el clúster, a través de la investigación acción-participación con la cual se pretende mejorar su realidad productiva de la mano de los mismos actores-empresarios.

Con el fin de orientar el análisis de estos casos se recurre a revisar varias perspectivas, todas de carácter evolutivo que hablan de la introducción de dinámicas y cambios, en la medida que avanza la experiencia asociativa de un grupo de empresas y otros entes, como fenómeno. La idea central es el cambio paulatino del fenómeno, pasando por distintos estados, que se pueden caracterizar con referencia a otras experiencias de cambio, en aspectos relacionados con los entornos interno y externo de cada organización, o de la red que conforman.

\section{Perspectivas para el análisis}

U na primera perspectiva teórica proviene de la evolución de las redes. Desde este punto de vista una red de entidades, es definida como un conjunto de elementos (las entidades: empresas, formas productivas, ideas, tecnologías, agentes, etc.) y las relaciones entre ellos, colocando el énfasis en las relaciones o interconexiones específicas y directas entre los elementos. Estas conexiones se expresan como interacciones entre agentes, modalidades de tecnología, formas de organizaciones, formas de competencia, contratos, reglas y normas de decisión, formas de procesamiento de información y de producción de conocimiento que pueden visualizarse como un espacio de conexiones (Potts, 2000). Como ellos requieren de

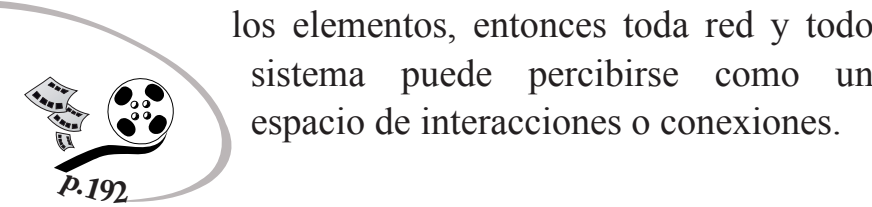

El concepto de red social está descrito por Faust, (2002) es una red social, comienza con la participación de los llamados actores sociales los cuales se vinculan entre ellos y con el exterior de diferentes maneras. Precisamente la importancia de las redes sociales radica en el estudio de la interacción de esos actores sociales. Las personas basan sus relaciones en amistad, parentesco, vecindad, profesión y otras más; por estas relaciones circulan información, asistencia, recursos, consejos y otros contenidos y pueden tener como requisitos para su existencia la comunicación, el respeto, la retribución o la lealtad entre los miembros de la red.

Una red social consiste en esencia en dos elementos, una población de actores y por lo menos una relación medible, definida para cada par de actores. Estos pueden ser 
"Una red social se interpreta básicamente por la información relacional que contiene; a diferencia de los modelos que se han trabajado tradicionalmente en las ciencias sociales y del comportamiento, en los cuales los atributos de las unidades independientes son el tema central de análisis". entidades sociales con cualquier nivel de agregación (personas, colectividades, organizaciones $\mathrm{o}$ países). Las relaciones pueden comprender cualquier acción, actividad, transacción, obligación, sentimiento $\mathrm{u}$ otro tipo de conexiones entre pares o entre grupos de actores.

Una red social se interpreta básicamente por la información relacional que contiene; a diferencia de los modelos que se han trabajado tradicionalmente en las ciencias sociales y del comportamiento, en los cuales los atributos de las unidades independientes son el tema central de análisis. Una red social requiere por tal razón de modelos que ayuden a representar los nexos entre las entidades sociales, así como de métodos que permitan estudiar sus propiedades relacionales. Faust, (2002) considera que la clave del análisis de redes sociales es la conceptualización de los fenómenos como hechos relacionales.

En las siguientes líneas se presentan las características fundamentales del análisis de redes según Rodríguez, (1995): dada la importancia central de la interacción entre unidades interactuantes, los actores y sus acciones son vistos como interdependientes, en lugar de independientes y autónomos; las uniones y relaciones entre actores, son canales de transferencia y distribución de recursos materiales o inmateriales; cuando se analizan individuos, se ve el modo estructural como un espacio que provee oportunidades o limitaciones para la acción individual; la estructura es definida como pautas relacionales relativamente permanentes.

Dentro de los principios analíticos de las redes sociales tenemos que: las uniones son normalmente asimétricas y recíprocas y difieren en contenido e intensidad; los lazos

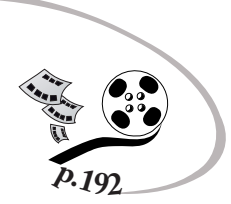
unen a los actores directa o indirectamente; los puntos de una red no tienen por qué ser personas individuales, pueden considerarse como grupos, estados, etc., o conjuntos de relaciones

clusters u otras redes; las uniones asimétricas y las redes complejas distribuyen recursos escasos o crean recursos nuevos de forma diferencial; las posiciones estructurales son a su vez recursos; las posiciones estructurales no son inamovibles; las redes estructuran actividades colaborativas o competitivas entre actores para asegurar recursos escasos o para crear recursos nuevos.

En la investigación y las observaciones sobre las aglomeraciones empresariales, se tiene el interés de encontrar no sólo la estructura de las relaciones entre los actores sociales vinculados a la actividad productiva, sino además el contenido de dichas relaciones y más específicamente el contenido relacionado con el trabajo y el conocimiento, en razón a que se intenta intervenir y participar en la transformación de la actividad productiva modificando los contenidos de trabajo y conocimiento que manejan actualmente los actores. A través de las relaciones fluye una serie de contenidos dependiendo el tipo de relación analizada.

Rodríguez (1995: p23) define los tipos más comunes de relaciones y contenidos:

¿ Relaciones transaccionales: intercambio de control sobre elementos físicos o simbólicos. (Regalos, compras, ventas, etc.).

Relaciones de comunicación: transmisión de mensajes.

$\downarrow$ Relaciones de penetración de bordes o fronteras: uniones entre subcomponentes en común.

\elaciones instrumentales: comparten el objetivo de asegurar un contenido o recurso valioso como mercancías valiosas, servicios, información a través de trabajo, consejo político, etc.

Relaciones sentimentales: expresión de sentimientos entre actores.

Relaciones de poder y autoridad: derechos y obligaciones de los actores para mandar y obedecer en organizaciones.

¿ Relaciones de parentesco: intervienen factores familiares, de amistad y compadrazgo. 
Esta concepción de red se apoya en la idea de difusión de un contenido en una red social; a manera de ejemplo, se reconoce que la innovación como conocimiento aplicado y comercializado, o el conocimiento en general, depende para su difusión en una comunidad, de la existencia de actores marginales, intermediarios y puentes (gate-keepers) que mantienen conexiones con sistemas más amplios y que por lo tanto integran elementos distantes dentro de una estructura más compleja.

Un objeto o fenómeno complejo, puede describirse y analizarse desde la perspectiva de los elementos y las conexiones. Y más allá, pueden seguirse los cambios en el fenómeno al identificar y analizar los cambios en los elementos y en las conexiones. De la misma manera, pueden producirse cambios en el fenómeno complejo o en el sistema (sistema de sistemas, propiedad hologramática de los sistemas), al intervenir sobre las relaciones o conexiones entre elementos o en los elementos mismos. Este es el fundamento del estudio de la dinámica de las redes, la jerarquía (Bertalanffy, 1962) y la emergencia.

La segunda perspectiva está basada en la evolución de la política que hace a su vez evolucionar las redes y la asociatividad al promover ambientes propicios para ésta.

Lall y Teubal (1998) plantean, la existencia de una diversificación de políticas públicas -no simplemente de exportaciones- para promover la entrada de las firmas en actividades que involucren tecnologías complejas (desarrollo tecnológico e innovación a partir de incorporación y generación de conocimiento tanto individual en el ámbito de cada empresa como colectivo vía asociatividad). Esta diversidad de políticas consiste en mezclar y ensayar políticas no solo transversales (i.e. orientadas a mejorar el funcionamiento de los mercados de factores sin favorecer en particular algún sector específico de la economía, como por ejemplo generar bienes no rivales como la estabilidad financiera y jurídica; entorno a la libre competencia, etc.) sino también de corte horizontal (i. e. actividades seleccionadas que se dirigen hacia actividades en donde no existen mercados, o que presentan grandes fallas, p. ej. actividades de investigación y desarrollo) y políticas verticales (i. e. provisión eficiente de los factores específicos que a nivel microeconómico, condicionan las capacidades y el desem-peño de las firmas, clusters y sectores e industrias específicas en el mercado nacional e internacional).

La políticas horizontales en CTI (ciencia, tecnología e innovación) son las que se ocupan de promover actividades socialmente deseables en un amplio espectro de sectores industriales y de tecnologías, incluyendo la I+D clásica en las empresas, la transferencia de tecnología, la absorción y la difu-sión, ciertos tipos de infraestructura tecnológica y otras funciones. (Teubal, 1996a), (Teubal, 1996b). Contrastan con las políticas de apoyo a sectores o industrias seleccionadas, a industrias nacientes o a tecnologías específicas, las políticas verticales en CTI.

Se acota que la existencia de políticas horizontales no sólo es congruente con las políticas y los programas orientados -políticas verticales-, sino que el desarrollo de programas horizontales puede conducir a un conjunto de programas más selectivos y focalizados como resultado del coaprendizaje y co-evolución, tanto de parte de quienes reciben los incentivos como por las agencias gubernamentales encargadas de ejecutar las políticas (Teubal, 1998). De aquí se desprende además que, la formulación y ejecución de políticas trae como consecuencia la evolución conjunta y aprendizaje contínuo y también conjunto de los agentes que intervienen.

Teubal (1998) hace la reflexión que, el atractivo de las políticas horizontales de CTI, en comparación con las políticas dirigidas, radica en la posibilidad de implementarlas sin tener, por parte de los agentes que las formulan, fuertes capacidades iniciales de política, pero si una capacidad inicial para aprender. Lo anterior, debido en parte a la posibilidad de dividir esfuerzos entre un conjunto de proyectos distribuidos en el tiempo, en contraste con las capacidades de coordinación que exige un pequeño número de inversiones grandes.

Se establece, a partir de la dinámica evolutiva entre formulación, ejecución y evaluación de las políticas horizontales y la formulación, ejecución y evaluación 
de políticas verticales o enfocadas, un ciclo de políticas tecnológicas o de CTI con fase inicial y fase madura. Fase inicial caracterizada por la búsqueda y logro de aprendizajes tanto por parte de los agentes de política como por los ejecutores y empresas. Tal perspectiva del aprendizaje abarca la I+D y la innovación en empresas y otras alternativas tecnológicas críticas para la reestructuración y adaptación de las mismas, como la transferencia de tecnología, la introducción de nuevas prácticas y técnicas de gestión y la I+D pre-competitiva, basada en la cooperación entre empresas. La fase madura, enfocada sobre la solución a fallas de mercado. Teubal (1998:9) cita como falla del mercado, la falla en la adopción de nuevas prácticas relacionadas con la innovación además de las fallas tradicionales del mercado.

El éxito en lograr una transición entre la primera y la segunda fases o etapas de esta dinámica evolutiva de la política de CTI, radica en adoptar una estrategia de aprendizaje durante la fase inicial y encarar la reestructuración de las políticas en la fase final. Requiere a su vez, el desarrollo de capacidades dentro de los entes gubernamentales, la formulación de políticas que vayan más allá de los incentivos y la adopción de una perspectiva evolutiva (Teubal, 1998).

Los aprendizajes que expresan la co-evolución entre agentes gubernamentales encargados de formular e implantar la política de CTI y los ejecutores de proyectos en las empresas y otras entidades generadoras o difusoras de conocimiento, están relacionados de manera explícita durante la primera etapa inicial, con elementos del aprendizaje para la innovación.

Los componentes que pueden considerarse en el apren- dizaje para la innovación son los siguientes (Pettigrew \& Fenton, 2000) (Teubal, 1998): Inteligencia de mercados e identificación de oportunidades de negocio o demandas sociales especificas; búsqueda de información tecnológica; identificación de agendas de investigación y de proyectos de $\mathrm{I}+\mathrm{D}$ e innovación, nuevos modelos de negocio; preselección, evaluación y selección de proyectos; identificación e integración del financiamiento para las agendas y proyectos; gestión de alianzas y redes con actores; gestión del proceso de innovación hasta el posicionamiento en el mercado del producto o servicio; transición a una organización que aprende, creadora de conocimiento (Nonaka \& Takeuchi, 1995).

Aquí se hace énfasis sobre el aprendizaje organizacional. Los aprendizajes más importantes que deben lograrse en la primera etapa de evolución de la política de CTI, son los que llevan a la organización (empresas y agentes gubernamentales en co-evolución) a cambios en estrategia, estructura y cultura que se expresan a su vez en cambios internos en el personal, los procesos y las tecnologías en uso. (Cooper, 1998)

En resumen, el paso de una etapa evolutiva inicial a una etapa madura en la implementación de políticas de CTI, está marcado por la detección de cambios en los agentes gubernamentales y en los ejecutores; cambios relacionados con aprendizajes que los facultan para la innovación y tienen que ver con aprendizaje organizativo.

Otra perspectiva de los aprendizajes y su relación con los impactos de las acciones que se ejecutan, puede verse en (Tassey, 2003), quien describe los resultados e impactos que se logran con el financiamiento a CTI, sobre una base de tiempo prolongado, Figura 1. Los clasifica en

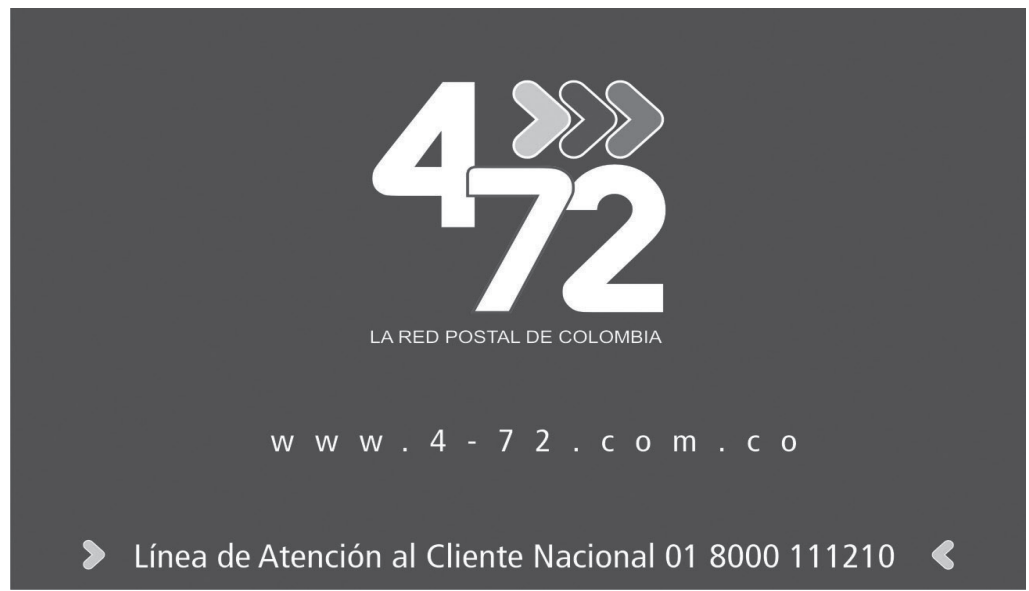




\section{Figura 1}

Etapas y tiempos para alcanzar impactos

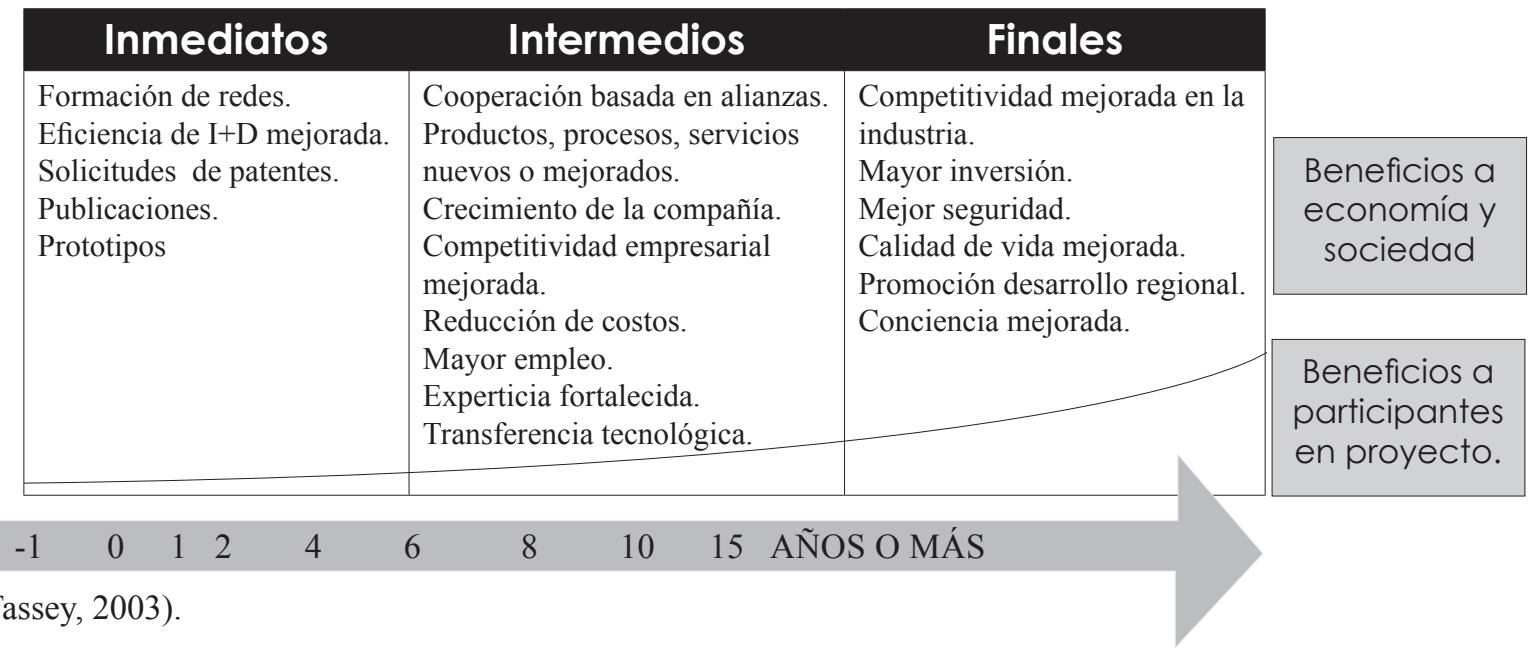

Fuente: tomado de (Tassey, 2003).

Se puede observar en la figura 1, que los beneficios esperados de los aprendizajes o impactos inmediatos e intermedios alcanzan mejor a los participantes en el proyecto y que los impactos y aprendizajes finales logran llegar a la sociedad y la economía. Para Tassey existe una base empírica de experiencias en financiación de proyectos en el NIST, que muestra que la política puede impulsar la aparición o emergencia de cooperación basada en alianzas, en una fase intermedia de la evolución de la misma, de lo cual se infiere que los incentivos y mecanismos que aporta el entorno de política en ejecución juega un papel importante en la conformación de redes y asociatividad, pero también que dicho entorno de política debe coevolucionar para producir los mecanismos e incentivos que se requieren, para producir tales efectos.

En resumen, analizar las experiencias de redes de negocios en los dos casos implica dos enfoques: la perspectiva de las redes para analizar lo que sucede en la aglomeración y la perspectiva de política evolutiva para describir lo que sucede en el entorno y que influye sobre cada experiencia.

\section{Metodología}

$\mathbf{P}$ ara desarrollar la investigación, en los dos casos, el autor participó como investigador en los dos ejercicios que se mencionan; durante cuatro años, cada uno de manera simultánea y en contratos sucesivos pagados o financiados por la universidad, por los actores empresarios o por entes públicos y privados de apoyo. En cada caso se actuó desde una universidad donde es una política la contratación de investigadores externos. En ambos casos, se actuó a través de un grupo de investigación amplio, multidisciplinario que desarrollaba esta tarea con la misión de realizar investigación socialmente útil. De aquí que se seleccionó la metodología de investigación-acción participativa, como forma de interactuar con los actores de las aglomeraciones que se estaban conformando.

Esta metodología comporta en su centro la idea propuesta por uno de sus iniciadores de que sólo se conoce el fenómeno o el problema cuando se intenta transformarlo, distinta a la posición de la investigación sobre objetos o sujetos independientes del investigador. De otra parte se anota que no fue planeado desde el inicio de la investigación, que esta fuera una investigación longitudinal, pero el tiempo de interacción con los actores empresarios y las observaciones, recomendaciones, ejecución de distintos proyectos participativos, etc., que se llevaron a cabo en cuatro años, muestran hallazgos interesantes que no es posible dejar pasar sin reflexionar sobre ellos, a fin de obtener aprendizajes y orientaciones para experiencias posteriores que están aflorando en el país a nivel central y en las regiones. Además, muestra que para analizar experiencias de procesos de cambio social lento como el que se presenta en la formación de aglomeraciones, asociatividades y clusters, no bastan los estudios transversales ni las referencias a otros estudios en otros países, sino que es necesaria la observación paciente e inteligente de cómo evoluciona el fenómeno en el entorno local. 
Los dos casos que se comparan, pertenecen, el primero a la conformación de la red de negocios de empresas de software y servicios asociados de Bogotá y Cundinamarca y el segundo a la intervención en el conglomerado de empresarios y artesanos de metales, piedras preciosas y joyería de la localidad de La Candelaria en Bogotá. Para el primer caso, se partió de la elaboración participativa de las orientaciones estratégicas para el grupo de empresas que iniciaron el ejercicio de asociatividad y posteriormente se acompañó la formulación y desarrollo de los proyectos asociativos resultantes, tales proyectos fortalecen la asociatividad a partir de la satisfacción de necesidades detectadas por los mismos actores y pueden agruparse en proyectos para desarrollar capacidades, proyectos para implementar procesos compartidos y proyectos para investigar y desarrollar productos $\mathrm{y}$ servicios en conjunto. Para el segundo caso, se observó la emergencia de un método de intervención, luego del primer año de trabajo, tal método de intervención se apoyó en conceptos de desarrollo de la competitividad presentados por Porter, e intentó fortalecer las capacidades individuales y colectivas, desarrollar conexiones y asociatividad, lo mismo que mejorar procesos técnicos y de gestión y desarrollar productos y mercados nuevos. Se analiza la dinámica de las dos experiencias a la luz de las perspectivas evolutivas que se han descrito arriba. Repitiendo la perspectiva de las redes para analizar lo que sucede en la aglomeración y la de política evolutiva para describir lo que sucede en el entorno y que influye sobre cada experiencia.

\section{Resultados}

$\mathbf{L}_{\mathrm{r}}^{\mathrm{o}}$ os hallazgos que se mencionan a continuación, son el resultado de la reflexión sobre los procesos ejecutados dentro de los dos casos y no sobre resultados específicos de los objetivos perseguidos por los proyectos que se ejecutaron en cada caso. Es una reflexión sobre las etapas del proceso de asociatividad, que resultan de la observación por cuatro años de los eventos sucedidos, los actores que intervinieron, hechos relevantes que muestran un patrón que puede ser tenido en cuenta en ulteriores experiencias. Si se quiere es un meta-análisis de las experiencias vistas en conjunto, con el fin de obtener de allí recomendaciones y visiones de lo que puede suceder en ejercicios asociativos. No tiene pretensiones de previsión, pero sí de convertirse en referente para desarrollos subsiguientes.

Las dos experiencias muestran distintos atributos, al analizarlas a partir de la óptica de las redes. Por este enfoque se analizan aspectos como el ámbito interno en el que se desarrolla la asociatividad; la emergencia de especialización vía conocimiento; la generación de relaciones, el papel de las acciones dinamizadoras. Por su parte, los aportes del entorno se analizan desde la perspectiva de la evolución de una política de incentivos.

Una primera observación, se relaciona con la preexistencia o no de relaciones entre las empresas o empresarios. En el primer caso de las empresas de software, los gerentes de dichas empresas comenzaron a establecer relaciones para comunicar sus intereses en asociarse, esto a partir de un contexto en el que un empresario líder que había tenido con los demás relaciones de trabajo, empieza a aglutinarlos invitándolos a reunirse; mientras que en el segundo caso, los artesanos y empresarios de joyería, ya habían desarrollado relaciones de trabajo conformando pequeños grupos no formales, que se distribuían el trabajo $y$ en el que varios individuos soportaban las exigencia de un pedido solicitado a uno de ellos. Las empresas en el primer caso están formalizadas, mientras que en segundo se han formalizado solo algunas de ellas. Si se acepta que las relaciones para ejecutar trabajo en común son más complejas que las conexiones para comunicar intereses y conocerse, el grupo de empresas de software comenzó el ejercicio con actividades de comunicación más sencillas. Mientras que el grupo de empresas de software comienza a sentir la necesidad de tener una orientación, objetivo común, etc. y para eso busca asesoría externa; el grupo de artesanos y empresarios de joyería, es abordado por personal de la universidad que quiere brindar apoyo para identificar problemas y buscar soluciones conjuntamente. En realidad la universidad buscaba también validar los hallazgos de un estudio, previo, descriptivo de la actividad productiva en la localidad. Aquí hay una diferencia importante, la motivación con la que el grupo de empresarios inicia el proceso; en un caso, la iniciativa parte de los propios empresarios mientras que en el otro, parte de la organización de apoyo ( la universidad).

En el primer caso, los recursos para el ejercicio asociativo fueron suministrados en parte por los empresarios y en 
parte por entes de apoyo gubernamentales; para el segundo caso la universidad suministró los recursos para las actividades iniciales a partir del pago de honorarios a investigadores. Puede decirse que el entorno representado en dos componentes: el institucional expresado en las regulaciones, normas e incentivos y el organizacional representado en las organizaciones de apoyo, brindó -no sin dificultadesrecursos, e introdujo políticas que favorecieron el inicio del ejercicio asociativo en ambos casos; pero que dicho inicio implicó la presencia de consultores o investigadores externos (consejeros técnicos, extensionistas, agentes actualizadores) -también en ambos casos-. Se analiza que durante una primera etapa dichos entes externos, apoyaron la incorporación de información nueva y facilitaron procesos de orientación e identificación de necesidades, en los dos casos, a través de agentes externos al proceso asociativo.

Puede inferirse, que en una primera etapa, provino del entorno la información y el conocimiento, que fue asimilado y transformado por las empresas y empresarios en planes orientadores y proyectos conjuntos de ejecución posterior, más independiente en el primer caso y apoyada con ayuda externa en el segundo caso. Las empresas y empresarios se asimilan metafóricamente a células que van formando un núcleo de conocimiento nuevo, tanto en aspectos de asociatividad experiencial, como de gestión en el primer caso y de gestión y producción en el segundo caso.

La primera etapa que se puede deducir, va hasta la ejecución de proyectos conjuntos para el primer caso o hasta la ejecución de actividades conjuntas en el segundo caso. Se distingue proyecto de actividad en la intensidad de coordinación, uso de recursos y límites de tiempo entre uno y otro. En el primer caso (empresas de software), los proyectos tienen objetivos más complejos de realizar, requieren mayor coordinación y comunicación, se enfrentan a exigencias del financiador o del cliente por calidad y cumplimiento de costos y tiempos; en el segundo caso (empresarios joyeros) las actividades son menos complejas, se orientan a intereses comunes, son en parte organizadas por los propios empresarios que requieren apoyo. En ambos casos constituyen acciones dinamizadoras que aumentan la necesidad de información y conocimiento en las empresas, lo que las hace más receptivas a entrar en contacto con otros entes externos para adquirir dicha información y conocimiento. Por su parte la generación de confianza se da en la acción. La repetición de comportamientos que reflejan compromiso, lo que a su vez demuestra entendimiento de requisitos individuales y colectivos y calidad en la comunicación, se genera y fortalece entre los empresarios como personas que representan a su empresa al realizar las acciones dinamizadoras representadas por los proyectos conjuntos.

Los proyectos que facilitan la interacción y el aprendizaje, se relacionan en el primer caso, con desarrollo de nuevas capacidades en las empresas (preparación para homologar normas internacionales de producto o de proceso, dominio de nuevos lenguajes de programación para la web, establecimiento de alianzas entre las mismas empresas vía negocios, aprendizajes sobre la gestión de proyectos), en el desarrollo de servicios conjuntos o de proyectos cooperativos para satisfacer demandas de un cliente (aseguramiento de calidad de datos, trazabilidad de datos, implementación de soluciones en procesos de otras empresas cliente); en la implantación de procesos conjuntos o compartidos entre las empresas (procesos de mercadeo, integración de soluciones, procesos de comercialización, procesos de capacitación) y finalmente, en el desarrollo de negocios entre las empresas de la red.

En el segundo caso, de los artesanos y empresarios de joyería; las actividades conjuntas se orientan a mejora de procesos técnicos, entendimiento de mercados nicho, incorporación de habilidades de diseño, sensibilización para la asociatividad, fortalecimiento de los grupos o redes de trabajo.

El análisis de esta actividad en los dos casos muestra que los proyectos comunes, además de facilitar el aprendizaje en la acción (learning by doing), aumentan la necesidad de relaciones entre los mismos ejecutores y con otros entes del entorno que los apoyan, los guían y les transfieren conocimiento. A su vez, el grupo de empresas 
y empresarios, comparte su conocimiento del negocio y los problemas y oportunidades relacionadas, con los agentes externos. Los proyectos conjuntos son una forma de intervención o auto-intervención en las relaciones o conexiones de las redes, que como se vio arriba, modifican el sistema completo y lo hacen movilizarse en evolución hacia adelante al aumentar en complejidad el entramado de conexiones y los contenidos que fluyen por ellas.

Una segunda etapa de evolución de la aglomeración de empresas y empresarios, puede describirse hasta la emergencia o aparición de organismos especializados en la red. Aquí se muestran diferentes los dos casos analizados; mientras que el grupo de empresas de software logra integrar intereses y recursos propios para generar y financiar nuevos organismos de la red especializados, por ejemplo en comercialización y en promoción de la
"Los proyectos comunes, además de facilitar el aprendizaje en la acción (learning by doing), aumentan la necesidad de relaciones entre los mismos ejecutores $y$ con otros entes del entorno que los apoyan, los guían y les transfieren conocimiento". innovación- si se extiende la I+D más allá de sí misma abarcando el lanzamiento al mercado de nuevos productos y servicios. Por su naturaleza, la innovación es un producto social, en el sentido que implica la integración de ideas, esfuerzos, recursos y tecnologías de múltiples fuentes, en sí misma implica también la administración de un tejido de relaciones que la hacen posible; no puede darse sino a través de la acción en red y de la comunicación y conexiones con múltiples agentes internos de las empresas o del conglomerado de empresas y agentes externos a este conglomerado, incluyendo agentes extranjeros.

Por esta razón surge la hipótesis que, la evolución de la asociatividad entre empresas en la forma de redes de negocios se ve acelerada o fortalecida a partir de las acciones de generación de innovaciones o de su gestión. La innovación coloca a las empresas y a la red de cara al mercado $\mathrm{y}$ en relación estrecha con el cliente y con las demandas. Pero esta innovación que genera la red de empresas no puede darse si cada una de ellas no desarrolla capacidades en el mismo sentido, precisamente por esto el organismo de promoción de la I+D creado en el caso de empresas de software, tiene como tarea inicial principal fortalecer las capacidades para la innovación en las empresas de la red.

Continuando con el símil de la célula, los organismos especializados que aparecen en la red en la segunda etapa, expresan en sí mismos la enucleación de conocimiento y experiencia, más acentuado, si el organismo se especializa en promover y desarrollar innovaciones, lo cual favorece el aumento de la densidad de conexiones entre empresas de la red y con el entorno.

Las dos experiencias siguen su curso. De aquí en adelante se puede prever una evolución hacia distintos escenarios de la red. En este documento solo se describe uno de esos escenarios, que por lo tanto es hipotético y marca la tercera etapa evolutiva en la que debe invertirse tiempo y esfuerzo y que comporta un escenario apuesta. En esta etapa se prevé la profundización de las prácticas de innovación ( $\mathrm{I}+\mathrm{D}$ más ingreso a mercados) en las empresas participantes de la red. Prácticas de innovación que facilitarán e impulsarán el aumento de complejidad en las relaciones, propiciando la emergencia de estructuras promoción de $\mathrm{I}+\mathrm{D}$ en la red de empresas, comporta
la introducción de un nuevo tipo de práctica, -la de la

En este punto debe hacerse una reflexión sobre el tipo el caso de las empresas de software, se dan dos tipos de la I+D en las empresas y de desarrollo de proyectos conjuntos de $\mathrm{I}+\mathrm{D}$. El segundo tipo ha resultado con mejores perspectivas de desarrollo que el primero. La de organismos nuevos que pueden aparecer en la red. En 
organizativas nuevas, núcleos de actividad especializada con necesidad de nuevas interconexiones. Si se sigue la senda evolutiva, se profundiza en la diversidad; la selección la realiza el mercado y la competencia y la reproducción se dará para los ganadores en la competencia.

Se destaca el papel de la gestión de la innovación tanto la conjunta como la de las empresas -en su atributo de producto social- como generadora de conexiones y por lo tanto de cambios; como núcleo de conocimiento y experiencia, como impulsadora del acercamiento íntimo con el mercado y el cliente. En este sentido las conexiones que se crean por el efecto de la actividad innovadora se establecen con entes que generan conocimiento e información, o con propietarios de activos de conocimiento, pero también con proveedores, con competidores, con agentes del mercado, con clientes, con entes de soporte y de incentivo y regulación, con agentes de capital de riesgo y estas relaciones deben buscarse y favorecerse.

Aquí cabe analizar el papel del entorno, representado en las organizaciones de soporte y en las políticas de incentivos y regulación. La evolución de las redes, expresadas en aglomeraciones de empresas ubicadas geográficamente, va de la mano con la evolución de la política que las favorece. (Teubal, 1998) destaca dos etapas en la evolución de la política de ciencia, tecnología

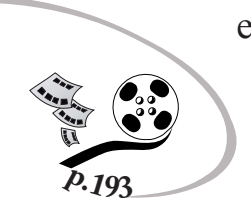
e innovación; una primera de aprendizajes por parte de los formuladores y también de los ejecutores de la política; vale decir, empresas, universidades, laboratorios, etc., una segunda de transición de políticas de corte horizontal, que sirven a todos los sectores e industrias para pasar a una de corte vertical, orientada a sectores e industrias específicas. Deja entrever una tercera etapa de madurez, que describe mejor (Tassey, 2003) donde la política entrega resultados e impactos en los sectores industriales y en la sociedad.
El análisis de los casos que se ha realizado, muestra que la política ha permitido el aprendizaje para las empresas de la red con algunas limitaciones, en el sentido, que la política se ha quedado corta en apoyar el ciclo completo de los proyectos, dado que ha hecho énfasis en la I+D sin llegar a la innovación que implica mercados. Pero de otra parte ha fomentado los grupos de investigación en las empresas, la formulación y ejecución de proyectos, la conformación de redes aún incipientes y sólo de investigadores, ha fomentado el aprendizaje de nuevas prácticas en la gestión de la tecnología, etc. Se puede decir que ha sido apropiada para la primera etapa de evolución de las redes, pero que deja ver falencias para una segunda y tercera etapas. El problema radica en que ambas instancias, la de los formuladores de política e incentivos y la de los ejecutores de proyectos en las universidades y empresas, deben sincronizar su aprendizaje y co-evolucionar al mismo ritmo. Eso implica también políticas experimentales, que se evalúen en tiempos cortos para tener oportunidad de corregir.

Un aspecto importante es que aún hay espacio para las políticas horizontales mientras comienzan a desarrollarse las de corte vertical en esta etapa de transición. La innovación, que moviliza las redes como producto social, requiere además de los incentivos para su desarrollo y entrega de nuevos productos y servicios, procesos y formas de negocio, de la construcción de una infraestructura industrial que facilite su difusión y uso. Dicha infraestructura se refiere a (Van de Ven, Polley, Garud, \& Venkataraman, 2008): arreglos institucionales para legitimar, regular y estandarizar la nueva tecnología; capacidad de recursos públicos para el conocimiento científico básico, mecanismos financieros y fuerza laboral competente; desarrollo de mercados, educación del consumidor y demanda; investigación y desarrollo propietario, manufactura, producción y sistemas de distribución por medio de firmas privadas para comercializar la innovación y generar riqueza. 


\section{Conclusiones}

a asociatividad es un fenómeno complejo, que no puede -analizarse de manera estática. Tiene dinámicas que deben estudiarse para identificar lo que la hace cambiar en el tiempo, pasar por fases o estados. Su evolución puede seguirse a partir de las relaciones, su densidad y sus contenidos. La intervención en ella, por parte de los propios actores participantes o de actores externos, implica trabajar sobre las relaciones más que sobre los actores.

El seguimiento de las experiencias asociativas muestra etapas que pueden distinguirse según el grado de complejidad que vayan alcanzando las relaciones. Un indicador de la transición entre etapas, puede ser la ejecución de proyectos conjuntos y otro, la aparición de organismos especializados.

Una actividad especializada que se expresa como práctica nueva entre las empresas que conforman la red de negocios o la aglomeración, es la innovación. Esta como proceso social, genera y es generada por conexiones sociales entre distintos agentes públicos y privados, requiere e impulsa el surgimiento y el detrimento de conexiones y la transferencia de contenidos, lo que redunda en el mantenimiento de algún estado de complejidad.

El entorno institucional influye sobre la asociatividad a partir de la política implícita o explícita sobre la innovación. Ella acelera o retarda la evolución de la red, es necesaria la co-evolución.

El seguimiento de las experiencias de asociatividad y redes de empresas en Colombia reviste gran importancia. Se están dedicando esfuerzos y recursos a desarrollarla en algunas regiones y en sectores, donde aparece espontáneamente orientada a algunos mercados-nicho, por lo cual hay oportunidad de intercambiar aprendizajes. Las políticas gubernamentales, las de ciencia y tecnología, pero también las fiscales deben tener en cuenta de manera explícita este fenómeno económico y propender por su desarrollo y madurez, procurando garantizar ambientes propicios a la innovación como actividad central y dinamizadora de estas redes.

\section{Bibliografía}

- Bertalanffy, L. (1962). General Systems Theory: A critical review. General Systems: 8, 1-20.

- Cooper, J. (1998). A multidimensional approach to the adoption of innovation. Management Decision, 36(8), 493-502.

- $\quad$ Nonaka, I., \& Takeuchi, H. (1995). The Knowledge-Creating Company. Oxford University Press.

- $\quad$ Pettigrew, A., \& Fenton, E. (2000). The innovating organization. London: Sage.

- $\quad$ Potts, J. (2000). The new evolutionary microeconomics: complexity, competence and adaptive behaviour. Massachusetts: Edward Elgar Publishing, Inc.

- $\quad$ Tassey. (2003). Methods for Assesing the Economic Impacts of Government R\&D. Planning Report 03- 1. National Institute of Standards and Technology. NIST.

- Teubal, M. (1996b). Implications of organizational learning for horizontal technology policies: An exploratory study. En S. a. Tsipori, Peripheral Countries of Europe. Routledge.

- Teubal, M. (1998). Marco de políticas para el financiamiento de la innovacion en economías en vías de industrialización. Mimeógrafo.

- $\quad$ Teubal, M. (1996a). $R \& D$ and technology policy at NICs as learning processes. World Development .

- Van de Ven, A. H., Polley, D. E., Garud, R., \& Venkataraman, S. (2008). The innovation journey. New York: Oxford University Press. 